\title{
Thijs PORCK, Old Age in Early Medieval England: a Cultural History
}

\section{Marthe Mensah}

\section{(2) OpenEdition}

\section{Journals}

Édition électronique

URL : https://journals.openedition.org/ccm/5094

DOI : $10.4000 / \mathrm{ccm} .5094$

ISSN : 2119-1026

\section{Éditeur}

Centre d'études supérieures de civilisation médiévale/Université de Poitiers

\section{Édition imprimée}

Date de publication : 1 septembre 2020

Pagination : 185-187

ISBN : 978-2-490783-06-9

ISSN : 0007-9731

\section{Référence électronique}

Marthe Mensah, «Thijs porck, Old Age in Early Medieval England: a Cultural History », Cahiers de civilisation médiévale [En ligne], 250-251 | 2020, mis en ligne le 01 septembre 2020, consulté le 09 décembre 2022. URL : http://journals.openedition.org/ccm/5094 ; DOI : https://doi.org/10.4000/ccm. 5094

\section{(c) (i) (3)}

Creative Commons - Attribution - Pas d'Utilisation Commerciale - Pas de Modification 4.0 International - CC BY-NC-ND 4.0

https://creativecommons.org/licenses/by-nc-nd/4.0/ 
Thijs Porck, Old Age in Early Medieval England: a Cultural History, Woodbridge, The Boydell Press (Anglo-Saxon Studies, 33), 2019.

Dans cet ouvrage abondamment documenté, Thijs Porck nous propose une étude de la vieillesse dans le haut Moyen Âge anglais, plus précisément entre 700 et 1100 .

Si rien ne prouve que les vieillards étaient rares au Moyen Âge et s'il est difficile de définir la vieillesse d'un point de vue chronologique ou fonctionnel, on peut y voir par contre un construit culturel, une représentation de la personne âgée fondée sur l'expérience réelle et la perception quotidienne de ce qu'elle est, mais aussi sur des topoï littéraires. C'est le « construit culturel de la vieillesse, tel que le reflète l'héritage culturel du haut Moyen Âge » qui constitue le thème principal de ce livre et qui s'attache à démontrer la complexité, la diversité et l'ambivalence des perceptions et représentations de cet âge. L'a. parle alors de conceptualisations culturelles, qui se répandent dans tout un groupe et qui font partie de la mentalité collective de celui-ci.

S'appuyant sur des sources nombreuses et variées, ilen étudie les spécificités historiques et culturelles dans une approche pluridisciplinaire qui variera selon les sources provenant pour la plupart d'une petite partie de la société seulement, noblesse ou clergé lettrés.

Le premier chapitre traite de la manière dont les Anglo-Saxons segmentaient la vie en différentes périodes, comment ils définissaient la vieillesse par rapport aux autres phases de la vie, et ce qu'une telle division révélait de leur façon d'appréhender la vieillesse.

Après une analyse critique des travaux de trois auteurs, Isabelle Cochelin (Medieval Life Cycles: Continuity and Change, I. Cochelin et K. E. Sмyth [dir.], Turnhout, Brepols [International Medieval Research, 18], 2013) et John Anthony Burrow (John Anthony Burrow, The Ages of Man, A Study in Medieval Writing and Thought, Oxford, Clarendon Press, 1988) l'a. nous propose les diverses conceptions des Anglo-Saxons concernant les différentes étapes de la vie. On y découvre ainsi plusieurs schémas. Le premier, binaire, oppose jeunesse et vieillesse et renvoie à la prédilection médiévale pour les antonymes. Ce n'est cependant pas le schéma le plus courant. Puis un schéma ternaire, inspiré par la parabole des trois Vigiles (Luc 12,36-8) et les récits apocryphes, pour aboutir à un découpage en quatre temps s'appuyant sur les quatre éléments, inspiré par Pythagore et défendu par Bède ( $\mathrm{VIII}^{\mathrm{e}} \mathrm{s}$.) dans son De temporum ratione et ensuite par Byrhtferth de Ramsey ( $\mathrm{x}^{\mathrm{e}}-\mathrm{XI}^{\mathrm{e}} \mathrm{s}$.) dans Enchiridion.

L'a. poursuit avec un découpage en cinq puis six étapes, s'appuyant ici également sur des précurseurs, respectivement Origène et Saint Augustin. On observera que, pour ce schéma des six âges de la vie, saint Augustin s'est basé sur l'analogie avec les six âges du monde dont le dernier, allant jusqu'à la fin des temps, correspond à la vieillesse qui, pour les Anglo-Saxons débute vers cinquante ans. L'a. met en évidence la variante introduite par Bède et Alcuin, héritier direct de Bède, qui relèvent un aspect de déclin, aetas decrepita, divisant ainsi la vieillesse en deux parties : senectus et aetas decrepita.

Dans les deux chapitres qui suivent, l'a. souligne l'ambivalence des représentations de la vieillesse : considérations de sagesse, représentations négatives de décrépitude.

Se fondant sur des homélies et des commentaires bibliques, d'une part, et d'autre part, sur la poésie sapientielle, représentatifs de la société, l'a. recense les aspects positifs du grand âge. Le senior est le sage, il a un rôle social et transmet ses connaissances, nées de l'expérience, bonne ou mauvaise. Témoin du passé, il est le gardien de la mémoire. L'a. évoque successivement le rôle social de ce vieillard et la considération dont il est l'objet, mais remarque que l'on attend également de lui une attitude pieuse et dévote sachant que sa fin est proche. Si cette attitude est fortement encouragée par les homélistes (Aelfric, Bède...) force leur est de constater que le vieillard est parfois un senex sine religione. La considération pour le senior est plus axée sur sa conduite durant cette période de vie que sur la durée de son existence.

Avec le troisième chapitre, l'a. aborde les aspects négatifs du grand âge. Il retient que les Anglo-Saxons étaient bien conscients que tous les vieillards ne jouissaient pas de ces aspects positifs et qu'ils savaient les nombreux désagréments qui accompagnaient la vieillesse. On trouve dans ce chapitre plusieurs poèmes, The Wanderer, The Seafarer, Elene qui illustrent les recherches et les observations de l'a. Vieillir était souvent synonyme de perte de capacités physiques, de maux divers, de perte de statut social et de relations affectives. La vieillesse s'accompagne alors de solitude et de tristesse. Par ces poèmes et avec les homélistes, l'a. nous rappelle que le déclin physique inévitable de l'homme est semblable à ce qui se produit pour toute chose ici-bas. Tout passe et les joies de ce monde sont éphémères. Il convient 
donc de s'en détacher et de se tourner vers ce qui ne passe pas, vers le Ciel.

L'a. conclut que les Anglo-Saxons abordaient la vieillesse avec peur et appréhension.

Les trois chapitres suivants concernent des groupes spécifiques de vieillards et décrivent la manière dont ils sont représentés dans le domaine culturel et artistique. Le chap. 4 traite de l'hagiographie et donc de la vie de saints anglais mais aussi de la transposition vernaculaire de textes latins. Ces textes utilisent des expressions et des topoï conventionnels visant à idéaliser le saint. Si les vies de saints anglo-saxons font peu référence à la vieillesse, lorsqu'elles en parlent, c'est sous forme de stéréotypes et de clichés : guides pleins de sagesse pour les plus jeunes membres de la communauté, décrépitude physique qui ne les épargne pas, ascétisme et grande piété malgré leur grand âge, miracles compensant leur ancienneté, attente joyeuse de la mort qui mettra fin aux aléas de la vieillesse. En fait, le grand âge révèle la sainteté de la personne et la puissance divine qui s'exprime par l'intermédiaire du saint. Celui-ci nous est présenté comme un modèle, mais un modèle difficile à imiter.

En réalité, ces saints d'un grand âge ne sont pas très différents des vieux guerriers que l'a. décrit au chapitre suivant. Comme eux, malgré leur âge, ils font preuve d'un courage héroïque inspiré par les valeurs auxquelles ils croient, celles que défend la poésie héroïque. Ils continuent à prendre part au combat, souvent en première ligne, avec une détermination sans failles, une loyauté indéfectible, tout comme le saint poursuit ses prières et ses dévotions jusqu'à sa mort. Les vieux guerriers sont remplis de sagesse et comme pour les saints on attend d'eux qu'ils conseillent les plus jeunes et transmettent leur expérience. Ce sont des modèles qui doivent stimuler ceux qui les entourent par leurs paroles mais surtout par leur comportement. Selon l'a., les vieillards pouvaient encore être des champions de la spiritualité comme de la guerre. Mais ce n'était pas toujours le cas et les Anglo-Saxons se détournaient du vieux guerrier qui ne conservait pas un rôle actif sur le champ de bataille. On retrouve le thème de la vieillesse active opposée à la vieillesse passive que l'a. développe dans le chap. 6 consacré au poème Beowulf. Il oppose deux modèles de rois, deux attitudes face à la vieillesse et la guerre : une attitude active et combattante, celle de Beowulf, et l'autre, passive, fondée sur la diplomatie, qui caractérise Hrothgar; l'une fondée sur la force physique qui prend à revers la décrépitude évoquée au troisième chapitre, l'autre axée sur la passivité et la diplomatie, que l'a. nomme passivité diplomatique. La question est alors de savoir si un roi âgé doit continuer à adopter une attitude hérö̈que ou si une approche passive et diplomatique est un comportement plus approprié. Pour l'a., il ne fait pas de doute que le poète présente Beowulf comme le modèle à suivre. Beowulf s'inscrit dans la lignée des guerriers des légendes germaniques et de la poésie héroïque vieil-anglaise pour lesquels la gloire et le bien de la tribu passent avant tout. Du point de vue de l'a., il s'agit là d'une nouvelle lecture du poème qui en fait un miroir de rois âgés et vient s'ajouter aux nombreuses lectures déjà réalisées.

Le dernier chapitre est consacré aux femmes âgées. L'a. note que les femmes sont faiblement représentées dans les écrits de l'époque et, lorsqu'elles le sont, elles appartiennent en général à la haute société. Il cite les exemples de reines douairières assumant un rôle politique, d'abbesses présentant un modèle religieux, et d'autres femmes qui, gardiennes de l'histoire locale et tribale, partagent leurs connaissances culturelles et parfois médicales ou simplement se chargent d'éduquer leurs petits enfants. Plutôt que d'analyser les aspects positifs et négatifs du grand âge, l'a s'oriente vers une approche plus sociale et constate que l'âge n'est pas synonyme de statut social inférieur.

On peut regretter avec l'a. une approche nécessairement partielle étant donné le peu de recherches menées à ce jour sur ce sujet et le peu de documents existants en dehors de correspondances personnelles ou de documents administratifs ou juridiques.

Cet ouvrage aborde donc successivement différents parcours de vie liés au grand âge et passe en revue divers aspects de la vieillesse dans la période considérée. L'a. s'attache à étayer son étude d'exemples très concrets tirés de sources diverses : arts visuels, documents écrits essentiellement, exégèse, hagiographie, poèmes, supports qui viennent appuyer et illustrer ses recherches et que le lecteur expert comme non expert appréciera. On note les nombreuses citations en latin ou en vieil anglais, pour lesquelles l'a. donne toujours une traduction en anglais moderne. On apprécie la synthèse faite par l'a. à la fin de chaque chapitre reprenant l'essentiel des éléments analysés, ainsi qu'une synthèse globale à la fin de l'ouvrage. On retrouve tout au long de l'ouvrage quelques auteurs privilégiés qui ont fait le Moyen Âge : Bède, né v. 672 a en quelque sorte ouvert la période étudiée par l'a., tandis qu'Aelfric, mort en 1010, en marque la fin. On regrettera cependant le chapitre peu développé concernant les femmes et la vieillesse, pour les raisons évoquées ci-dessus, mais qui laisse une piste de recherche ouverte pour cette thématique. L'a. propose également d'autres 
pistes de recherche qui pourront intéresser les futurs doctorants tout autant que les chercheurs.

Voilà donc une étude assez approfondie sur la vieillesse dans le haut Moyen Âge anglais qui sera un support de travail pour les spécialistes du Moyen Âge anglais mais aussi pour les chercheurs en sciences humaines et sociales, gérontologues et autres disciplines, intéressés par des éléments en lien avec cette période de la vie et de l'histoire.

Marthe MensaH Université de Reims 